\title{
Some results on $q$-harmonic number sums
}

\section{$\mathrm{Xin} \mathrm{Si}^{*}$}

*Correspondence: xsi@xmut.edu.cn School of Applied Mathematics, Xiamen University of Technology, Xiamen, P.R. China

\begin{abstract}
In this paper, we establish some relations involving $q$-Euler type sums, $q$-harmonic numbers and $q$-polylogarithms. Then, using the relations obtained with the help of $q$-analog of partial fraction decomposition formula, we develop new closed form representations of sums of $q$-harmonic numbers and reciprocal $q$-binomial coefficients. Moreover, we give explicit formulas for several classes of $q$-harmonic sums in terms of $q$-polylogarithms and $q$-harmonic numbers. The given representations are new.
\end{abstract}

MSC: 05A30; 65B10; 33D05; 11M99; 11M06; $11 \mathrm{M} 32$

Keywords: $q$-harmonic number; $q$-binomial coefficient; $q$-polylogarithm function

\section{Introduction and preliminaries}

Let $k, r, m_{1}, m_{2}, \ldots, m_{r}$ be positive integers and $p \in \mathbb{N}_{0}:=\{0,1,2, \ldots\}$ with $p+k>1$. The Euler type sums $W_{k}\left(m_{1}, m_{2}, \ldots, m_{r}, p\right)$ involving harmonic numbers and binomial coefficients are defined by the convergent series [1]

$$
W_{k}(\mathbf{m} ; p)=W_{k}\left(m_{1}, m_{2}, \ldots, m_{r} ; p\right):=\sum_{n=1}^{\infty} \frac{H_{n}^{\left(m_{1}\right)} H_{n}^{\left(m_{2}\right)} \cdots H_{n}^{\left(m_{r}\right)}}{n^{p}\left(\begin{array}{c}
n+k \\
k
\end{array}\right)}
$$

where $H_{n}^{(m)}$ stands for the $n$th generalized harmonic number defined by

$$
H_{n}^{(m)}:=\sum_{j=1}^{n} \frac{1}{j^{m}} \quad(n, k \in \mathbb{N}:=\{1,2,3, \ldots\})
$$

the quantity $w:=m_{1}+m_{2}+\cdots+m_{r}+p+k$ and the quantity $r$ are called the weight and the degree of (1.1), respectively. The empty sum $H_{0}^{(m)}$ is conventionally understood to be zero. When $m=1$, then $H_{n}:=H_{n}^{(1)}$ is called a classical harmonic number. As usual, we let $\{a\}_{k}$ be the $k$ repetitions such that

$$
W\left(a,\{m\}_{r}, b ; p\right)=W(a, \underbrace{m, \ldots, m}_{r}, b ; p) .
$$

(c) The Author(s) 2018. This article is distributed under the terms of the Creative Commons Attribution 4.0 International License (http://creativecommons.org/licenses/by/4.0/), which permits unrestricted use, distribution, and reproduction in any medium, provided you give appropriate credit to the original author(s) and the source, provide a link to the Creative Commons license, and indicate if changes were made. 
There are many results for sums of harmonic numbers with positive terms. For example, in [1], Xu et al. proved the result

$$
W_{k}\left(\{1\}_{2} ; 1\right)=\sum_{r=1}^{k}(-1)^{r+1}\left(\begin{array}{c}
k \\
r
\end{array}\right)\left\{\begin{array}{c}
3 \zeta(3)+\frac{H_{r}^{3}+3 H_{r} H_{r}^{(2)}+2 H_{r}^{(3)}}{3} \\
-\frac{H_{r}^{2}+H_{r}^{(2)}}{r}-\sum_{i=1}^{r-1} \frac{H_{i}}{i^{2}}+\zeta(2) H_{r-1}
\end{array}\right\} \quad(k \in \mathbb{N}) .
$$

Sofo also obtained many other identities involving harmonic numbers and central binomial coefficients. For instance, in [2], Sofo gave the following identity:

$$
W_{k}\left(\{1\}_{2} ; 0\right)=\frac{k}{k-1}\left(\zeta(2)-H_{k-1}^{(2)}+\frac{2}{(k-1)^{2}}\right) \quad(2 \leq k \in \mathbb{N})
$$

where $\zeta(p)$ stands for the classical Riemann zeta function defined by [3]

$$
\zeta(p):=\sum_{n=1}^{\infty} \frac{1}{n^{p}} \quad(\Re(p)>1)
$$

There are many works investigating sums of both harmonic numbers and binomial coefficients (see, for example, $[1,2,4-6]$ and the references therein).

If $k=0$ in (1.1), then

$$
W_{0}(\mathbf{m} ; p)=W_{0}\left(m_{1}, m_{2}, \ldots, m_{r} ; p\right):=\sum_{n=1}^{\infty} \frac{H_{n}^{\left(m_{1}\right)} H_{n}^{\left(m_{2}\right)} \cdots H_{n}^{\left(m_{r}\right)}}{n^{p}},
$$

which is just the classical Euler sums $S_{\mathbf{m}, \mathbf{p}}$ defined in [7], where $\mathbf{m}:=\left(m_{1}, m_{2}, \ldots, m_{r}\right)$. The study of Euler sums $W_{0}(\mathbf{m} ; p)$ was started by Euler. Euler's original contribution was a method to reduce double sums $W_{0}(p ; q)$ (or $S_{p, q}$ ) to certain rational linear combinations of products of zeta values. Examples for such evaluations, all due to Euler, are as follows:

$$
\begin{aligned}
& W_{0}(1 ; 3)=\frac{5}{4} \zeta(4), \quad W_{0}(1 ; 4)=3 \zeta(5)-\zeta(2) \zeta(3), \\
& W_{0}(2 ; 4)=\zeta^{2}(3)-\frac{1}{3} \zeta(6), \quad W_{0}(2 ; 5)=5 \zeta(2) \zeta(5)+2 \zeta(3) \zeta(4)-10 \zeta(7) .
\end{aligned}
$$

After that many different methods, including partial fraction expansions, Eulerian beta integrals, summation formulas for generalized hypergeometric functions and contour integrals, have been used to evaluate these sums. The relationship between the values of the Riemann zeta function and the classical Euler sums $W_{0}(\mathbf{m} ; p)$ (or $S_{\mathbf{m} ; p}$ ) has been studied by many authors (for example, see [7-16] and the references therein).

So far, surprisingly little work has been done on $q$-analogues of Euler sums. We begin with some basic notation. Let $q$ be a real number with $0<q<1$. The $q$-analogue of a nonnegative integer $n$ is defined as

$$
n_{q}=[n]_{q}:=\sum_{k=0}^{n-1} q^{k}=\frac{1-q^{n}}{1-q}
$$


For any real number $a$, put

$$
(a)_{0}:=(a ; q)_{0}=1 \quad \text { and } \quad(a)_{n}:=(a ; q)_{n}:=\prod_{k=1}^{n}\left(1-a q^{k-1}\right), \quad n \geq 1
$$

Let $n, m$ denote integers. Then the Gaussian $q$-binomial coefficient is defined by

$$
\left[\begin{array}{c}
n \\
m
\end{array}\right]_{q}:=\frac{(q)_{n}}{(q)_{m}(q)_{n-m}}=\frac{[n]_{q} !}{[m]_{q} ![n-m]_{q} !}
$$

where $0 \leq m \leq n$ and $[n]_{q} !=[1]_{q}[2]_{q} \cdots[n]_{q}$ with $\left[\begin{array}{c}n \\ 0\end{array}\right]_{q}=1$.

For non-negative integers $n, s$ and $m \in \mathbb{N}$, define $q$-analogues of harmonic numbers

$$
\zeta_{n}\left[m, q^{s}\right]:=\sum_{j=1}^{n} \frac{q^{s j}}{[j]_{q}^{m}}
$$

with the convention that $\zeta_{0}\left[m, q^{s}\right]:=0$. When $s=0$ and 1 , we use the following notations (see [17]):

$$
\left[H_{n}\right]:=\zeta_{n}[1]=\zeta_{n}[1,1]
$$

and

$$
\zeta_{n}[m]:=\zeta_{n}[m, 1], \quad\left[H_{n}^{(m)}\right]:=\zeta_{n}[m, q]
$$

Similar as in the definition of classical Euler sum $W_{0}(\mathbf{m} ; p)$ (or $\left.S_{\mathbf{m} ; p}\right)$, the $q$-analogues of Euler sum of index $\mathbf{m}:=\left(m_{1}, \ldots, m_{r}\right), \mathbf{s}:=\left(s_{1}, \ldots, s_{r}\right)$ with $p>1$ are defined by

$$
W_{0, t}^{(\mathbf{s})}[\mathbf{m} ; p]:=\sum_{n=1}^{\infty} \frac{\zeta_{n}\left[m_{1}, q^{s_{1}}\right] \zeta_{n}\left[m_{2}, q^{s_{2}}\right] \cdots \zeta_{n}\left[m_{r}, q^{s_{r}}\right]}{\left([n]_{q}\right)^{p}} q^{t n}
$$

where $s_{i} \in \mathbb{N}_{0}, m_{i} \in \mathbb{N}(i=1,2, \ldots, r)$ and $r, t \in \mathbb{N}$, the quantities $w:=m_{1}+m_{2}+\cdots+m_{r}+$ $p+k$ and $r$ are called the weight and the degree of $W_{0, t}^{(\mathbf{s})}[\mathbf{m} ; p]$, respectively. There are fewer results for sums of the type (1.7). Some related results for $q$-Euler type sums and related sums (e.g. $q$-L-function and $q$-multiple zeta values) may be seen in the works of [17-37] and the references therein. For example, in [17], Xu et al. gave the following identity:

$$
W_{0,1}^{(0)}[1 ; s]=\operatorname{Li}_{s+1}[q]+\frac{s}{2} \operatorname{Li}_{s+1}\left[q^{2}\right]-\frac{1}{2} \sum_{j=2}^{s-1} \operatorname{Li}_{j}[q] \operatorname{Li}_{s+1-j}[q], \quad 2 \leq s \in \mathbb{N}
$$

Examples for such evaluation are as follows:

$$
\sum_{n=1}^{\infty} \frac{\left[H_{n}\right]}{n_{q}^{2}} q^{n}=\mathrm{Li}_{3}[q]+\mathrm{Li}_{3}\left[q^{2}\right], \quad \sum_{n=1}^{\infty} \frac{\left[H_{n}\right]}{n_{q}^{3}} q^{n}=\frac{3}{2} \operatorname{Li}_{4}\left[q^{2}\right]+\mathrm{Li}_{4}[q]-\frac{1}{2} \operatorname{Li}_{2}^{2}[q]
$$

Furthermore, they proved the following conclusion: for positive integer $s \geq 2$, the quadratic sum $W_{0,1}^{(0,0)}\left[\{1\}_{2} ; s\right]$ and the cubic combination sum $W_{0,1}^{(0,0,0)}\left[\{1\}_{3} ; s\right]-3 W_{0,1}^{(0,0)}[1,2$; 
$s$ ] are reducible to linear $q$-Euler sums and to polynomials in $q$-polylogarithms. In particular, we have

$$
W_{0,1}^{(0,0)}\left(\{1\}_{2} ; 2\right)=\frac{7}{2} \mathrm{Li}_{4}\left[q^{2}\right]+2 \mathrm{Li}_{4}[q]-\frac{1}{2} \mathrm{Li}_{2}^{2}[q]-(1-q)\left(\operatorname{Li}_{3}\left[q^{2}\right]+\mathrm{Li}_{3}[q]\right) .
$$

The $q$-analogues of polylogarithm function $\mathrm{Li}_{m}[x]$ and linear $q$-Euler sum $W_{0, t}^{(s)}[m ; p]$ are defined by

$$
\begin{aligned}
& \operatorname{Li}_{m}[x]:=\sum_{n=1}^{\infty} \frac{x^{n}}{[n]_{q}^{m}}, \quad|x|<1, \\
& W_{0, t}^{(s)}[m ; p]:=\sum_{n=1}^{\infty} \frac{\zeta_{n}\left[m, q^{s}\right]}{[n]_{q}^{p}} q^{t n},
\end{aligned}
$$

where $t, m \in \mathbb{N}, s \in \mathbb{N}_{0}$ and $p>1$.

In this paper we will develop identities, closed form representations of $q$-harmonic numbers and reciprocal $q$-binomial coefficients of the form:

$$
W_{k, t}^{\left(s_{1}, \ldots, s_{r}\right)}\left[m_{1}, m_{2}, \ldots, m_{r} ; p\right]:=\sum_{n=1}^{\infty} \frac{\zeta_{n}\left[m_{1}, q^{s_{1}}\right] \zeta_{n}\left[m_{2}, q^{s_{2}}\right] \cdots \zeta_{n}\left[m_{r}, q^{s_{r}}\right]}{[n]_{q}^{p}\left[\begin{array}{c}
n+k \\
k
\end{array}\right]_{q}} q^{t n}
$$

for $p=0$ and 1 with $t=k$ and $k-1$. Here, $s_{i} \in \mathbb{N}_{0}, m_{i} \in \mathbb{N}(i=1,2, \ldots, r)$ and $k, r \in \mathbb{N}$. We show that the linear sums $W_{k, k}^{(m-1)}[m ; 1]$ is a rational linear combination of products of $q$-harmonic numbers and $q$-polylogarithms, and we give an explicit formula. We also provide explicit evaluations of quadratic sum $W_{k, k}^{(0,0)}\left[\{1\}_{2} ; 1\right]$ in a closed form in terms of $q$-polylogarithms, $q$-harmonic numbers and $q$-rational series. Furthermore, we prove that the cubic sum $W_{k, k}^{(0,0,0)}\left[\{1\}_{3} ; 1\right]$ is expressible in terms of $q$-polylogarithms, $q$-harmonic numbers and $q$-rational series. Letting $q$ approach 1 , we can find that the $q$-Euler type sum $W_{k, t}^{(\mathbf{s})}[\mathbf{m} ; p]$ converges to the classical Euler type sums $W_{k}(\mathbf{m} ; p)$, namely

$$
\lim _{q \rightarrow 1} W_{k, t}^{\left(s_{1}, \ldots, s_{r}\right)}\left[m_{1}, m_{2}, \ldots, m_{r} ; p\right]=W_{k}\left(m_{1}, m_{2}, \ldots, m_{r} ; p\right)
$$

Next, we prove a lemma which will be useful in the development of the main theorems.

Lemma 1.1 For positive integers $m, r, k$ and $r<k$, then

$$
\begin{aligned}
& \sum_{n=1}^{\infty} \frac{q^{m n}}{[n+r]_{q}^{m}[n+k]_{q}} \\
& \quad=\sum_{j=1}^{m-1} \frac{(-1)^{j-1}}{[k-r]_{q}^{j} q^{r j}} \mathrm{Li}_{m-j+1}\left[q^{m-j}, r\right]+\frac{(-1)^{m-1}}{[k-r]_{q}^{m} q^{r m}}\left\{\left[H_{k}^{(1)}\right]-\left[H_{r}^{(1)}\right]\right\}
\end{aligned}
$$

where the q-special function $\operatorname{Li}_{p}[x, a]$ is defined by

$$
\operatorname{Li}_{p}[x, a]:=\sum_{n=1}^{\infty} \frac{x^{n}}{[n+a]_{q}^{p}}, \quad|x|<1, p \in \mathbb{N}
$$


Proof By a simple calculation, the sum on the left-hand side of (1.12) is equal to

$$
\begin{aligned}
\sum_{n=1}^{\infty} \frac{q^{m n}}{[n+r]_{q}^{m}[n+k]_{q}}= & \sum_{j=1}^{m-1} \frac{(-1)^{j-1}}{[k-r]_{q}^{j} q^{r j}} \sum_{n=1}^{\infty} \frac{q^{(m-j) n}}{[n+r]_{q}^{m-j+1}} \\
& +\frac{(-1)^{m-1}}{[k-r]_{q}^{m-1} q^{r(m-1)}} \sum_{n=1}^{\infty} \frac{q^{n}}{[n+r]_{q}[n+k]_{q}}
\end{aligned}
$$

On the other hand, we note that, for $N>k>r \geq 1$ and $N, k, r \in \mathbb{N}$,

$$
\begin{aligned}
\sum_{n=1}^{\infty} \frac{q^{n}}{[n+r]_{q}[n+k]_{q}} & =\frac{1}{[k-r]_{q} q^{r}} \sum_{n=1}^{\infty}\left\{\frac{1}{[n+r]_{q}}-\frac{1}{[n+k]_{q}}\right\} \\
& =\frac{1}{[k-r]_{q} q^{r}} \lim _{N \rightarrow \infty} \sum_{n=1}^{N}\left\{\frac{1}{[n+r]_{q}}-\frac{1}{[n+k]_{q}}\right\} \\
& =\frac{1}{[k-r]_{q} q^{r}}\left\{\frac{1}{[r+1]_{q}}+\cdots+\frac{1}{[k]_{q}}-\lim _{N \rightarrow \infty} \sum_{j=r+1}^{k} \frac{1}{[N+j]_{q}}\right\}
\end{aligned}
$$

and

$$
\lim _{N \rightarrow \infty}(N+j)_{q}=\lim _{N \rightarrow \infty} \frac{1-q^{N+j}}{1-q}=\frac{1}{1-q}
$$

By using the definition of $q$-harmonic numbers, we have the relations

$$
\begin{aligned}
& {\left[H_{n}^{(m)}\right]=(q-1) \zeta_{n}[m-1]+\zeta_{n}[m], \quad m \in \mathbb{N},} \\
& {\left[H_{n}^{(1)}\right]=\left[H_{n}\right]+n(q-1) .}
\end{aligned}
$$

Combining (1.15), (1.16) and (1.18) yields

$$
\sum_{n=1}^{\infty} \frac{q^{n}}{[n+r]_{q}[n+k]_{q}}=\frac{1}{[k-r]_{q} q^{r}}\left\{\left[H_{k}^{(1)}\right]-\left[H_{r}^{(1)}\right]\right\}
$$

Substituting (1.19) into (1.14) yields the desired result. The proof of Lemma 1.1 is finished.

\section{Main conclusions and proofs}

In this section, we will give the main results of the present paper. Firstly, we establish a $q$ analog of partial fraction decomposition formula by the method of mathematical induction. Secondly, using the Jackson's $q$-integral, we prove some relations between $q$-Euler type sums, $q$-harmonic numbers and $q$-polylogarithms. Then we use the formulas obtained to evaluate several infinite series involving $q$-harmonic numbers.

It is clear that the conclusions which we present here can be seen as an extension of classical Euler type sums given by Sofo and Xu. Letting $q \rightarrow 1$, we obtain many well-known results which are given by Sofo and $\mathrm{Xu}$. 


\section{1 $q$-analog of partial fraction decomposition formula}

Theorem 2.1 For positive integer $m$ and real $x$ with $x \neq-1,-2, \ldots,-m$, the following identity holds:

$$
\prod_{i=1}^{m}\left(1-q^{x+i}\right)^{-1}=\sum_{i=1}^{m} \frac{q^{(1-m) x}}{1-q^{x+i}} \prod_{j=1, j \neq i}^{m} \frac{1}{q^{i}-q^{j}} .
$$

Notice that the term in the sum for $m=1$ is the empty product which is 1 , namely, when $m=1$, we set $\prod_{j=1, j \neq 1}^{1}\left(q^{i}-q^{j}\right)^{-1}:=1$.

Proof The proof is by induction on $m$. For $m=1$, we have $\prod_{i=1}^{1}\left(1-q^{x+i}\right)^{-1}=\left(1-q^{x+1}\right)^{-1}$, and the formula is true. For $m>1$, we proceed as follows. First assume that formula (2.1) holds for $m \leq k-1$, we note that

$$
\begin{aligned}
\frac{1}{\prod_{i=1}^{k}\left(1-q^{x+i}\right)} & =\frac{1}{1-q^{x+k}} \cdot \frac{1}{\prod_{i=1}^{k-1}\left(1-q^{x+i}\right)} \\
& =\sum_{i=1}^{k-1} \frac{q^{(2-k) x}}{\left(1-q^{x+k}\right)\left(1-q^{x+i}\right)} \prod_{j=1, j \neq i}^{k-1} \frac{1}{q^{i}-q^{j}} \\
& =\sum_{i=1}^{k-1} \frac{q^{(2-k) x}}{q^{x+i}-q^{x+k}}\left\{\frac{1}{1-q^{x+i}}-\frac{1}{1-q^{x+k}}\right\} \prod_{j=1, j \neq i}^{k-1} \frac{1}{q^{i}-q^{j}} \\
& =\sum_{i=1}^{k-1} \frac{q^{(1-k) x}}{1-q^{x+i}} \prod_{j=1, j \neq i}^{k} \frac{1}{q^{i}-q^{j}}-\frac{q^{(1-k) x}}{1-q^{x+k}} \sum_{i=1}^{k-1} \prod_{j=1, j \neq i}^{k} \frac{1}{q^{i}-q^{j}} .
\end{aligned}
$$

Then, by the induction hypothesis, we have that

$$
\begin{aligned}
& \frac{1}{\prod_{i=1}^{k-1}\left(1-q^{x+i}\right)} \\
& =\sum_{i=1}^{k-1} \frac{q^{(2-k) x}}{1-q^{x+i}} \prod_{j=1, j \neq i}^{k-1} \frac{1}{q^{i}-q^{j}}, \quad x \neq-1,-2, \ldots,-(k-1) .
\end{aligned}
$$

Setting $x=-k$ in the above equation, we deduce that

$$
\begin{aligned}
\frac{1}{\prod_{i=1}^{k-1}\left(1-q^{i-k}\right)} & =\frac{q^{k(k-1)}}{\prod_{i=1}^{k-1}\left(q^{k}-q^{i}\right)}=\sum_{i=1}^{k-1} \frac{q^{(k-2) k}}{1-q^{i-k}} \prod_{j=1, j \neq i}^{k-1} \frac{1}{q^{i}-q^{j}} \\
& =-\sum_{i=1}^{k-1} \frac{q^{k(k-1)}}{q^{i}-q^{k}} \prod_{j=1, j \neq i}^{k-1} \frac{1}{q^{i}-q^{j}} .
\end{aligned}
$$

Hence, we obtain

$$
\prod_{i=1}^{k-1}\left(q^{k}-q^{i}\right)^{-1}=-\sum_{i=1}^{k-1} \prod_{j=1, j \neq i}^{k} \frac{1}{q^{i}-q^{j}} .
$$


Substituting (2.4) into (2.2), we arrive at the conclusion that

$$
\prod_{i=1}^{k}\left(1-q^{x+i}\right)^{-1}=\sum_{i=1}^{k} \frac{q^{(1-k) x}}{1-q^{x+i}} \prod_{j=1, j \neq i}^{k} \frac{1}{q^{i}-q^{j}}
$$

The proof of Theorem 2.1 is completed.

Next, we give a $q$-analog of partial fraction decomposition formula.

Corollary 2.2 For integer $m>0$ and real $x$ with $x \neq\{-1,-2, \ldots,-m\}$, we have

$$
\prod_{i=1}^{m}[x+i]_{q}^{-1}=\sum_{i=1}^{m}(-1)^{i-1}[i]_{q}\left[\begin{array}{c}
m \\
i
\end{array}\right]_{q} \frac{q^{(1-m) x-\frac{i}{2}(2 m-1-i)}}{[m]_{q} !} \cdot \frac{1}{[x+i]_{q}}
$$

where $[x+i]_{q}:=\frac{1-q^{x+i}}{1-q}$.

Proof Multiplying $(2.1)$ by $(1-q)^{m}$ and using the definition of $[x+i]_{q}$, we obtain

$$
\begin{aligned}
\frac{1}{\prod_{i=1}^{m}[x+i]_{q}} & =\frac{1}{\prod_{i=1}^{m} \frac{1-q^{x+i}}{1-q}}=\sum_{i=1}^{m} \frac{q^{(1-m) x}}{\frac{1-q^{x+i}}{1-q}} \prod_{j=1, j \neq i}^{m} \frac{1}{\frac{q^{i}-q^{j}}{1-q}} \\
& =\sum_{i=1}^{m} \frac{q^{(1-m) x}}{[x+i]_{q}} \prod_{j=1, j \neq i}^{m} \frac{1}{\frac{q^{i}-q^{j}}{1-q}}
\end{aligned}
$$

We may rewrite the product on the right-hand side of (2.6) as follows:

$$
\begin{aligned}
\prod_{j=1, j \neq i}^{m} \frac{1}{\frac{q^{i}-q^{j}}{1-q}} & =\prod_{j=1}^{i-1} \frac{1}{-q^{j} \frac{1-q^{i-j}}{1-q}} \cdot \prod_{j=i+1}^{m} \frac{1}{q^{i} \frac{1-q^{j-i}}{1-q}} \\
& =\frac{(-1)^{i-1}}{q^{\frac{i}{2}(2 m-1-i)}} \cdot \frac{1}{[i-1]_{q} ![m-i]_{q} !}
\end{aligned}
$$

Combining (2.6) with (2.7), we may deduce the desired result. This completes the proof of Corollary 2.2 .

By using the definition of $q$-binomial coefficient in (1.6) and combining (2.5) with $m=$ $k, x=n(k, n \in \mathbb{N})$, we have the following expansion:

$$
\frac{1}{\left[\begin{array}{c}
n+k \\
k
\end{array}\right]_{q}}=\frac{[k]_{q} !}{\prod_{i=1}^{k}[n+i]_{q}}=\sum_{i=1}^{k}(-1)^{i-1}[i]_{q}\left[\begin{array}{c}
k \\
i
\end{array}\right]_{q} \frac{q^{(1-k) n-\frac{i}{2}(2 k-1-i)}}{[n+i]_{q}} .
$$

Similarly, using a similar argument, we can get

$$
\frac{1}{\left[\begin{array}{c}
n+k+r \\
k
\end{array}\right]_{q}}=[k]_{q} \sum_{i=1}^{k-1}(-1)^{i-1}[i]_{q}\left[\begin{array}{c}
k-1 \\
i
\end{array}\right]_{q} \frac{q^{(2-k)(n+r+1)-\frac{i}{2}(2 k-3-i)}}{[n+r+1]_{q}[n+r+1+i]_{q}}
$$

where $r \in \mathbb{N}_{0}$ and $2 \leq k \in \mathbb{N}$. 


\subsection{Identities for $q$-Euler type sums}

Theorem 2.3 For positive integers $m$ and $k$, the following identity holds:

$$
\sum_{n=1}^{\infty} \frac{\zeta_{n}\left[m, q^{m-1}\right]}{[n]_{q}[n+k]_{q}} q^{n}=\frac{1}{[k]_{q}}\left\{\begin{array}{c}
\operatorname{Li}_{m+1}\left[q^{m}\right]+(-1)^{m-1} \sum_{i=1}^{k-1} \frac{\left[H_{i}^{(1)}\right]}{[i]_{q}^{m}} q^{i} \\
+\sum_{j=1}^{m-1}(-1)^{j-1} \operatorname{Li}_{m+1-j}\left[q^{m-j}\right]\left[H_{k-1}^{(j)}\right]
\end{array}\right\}
$$

Proof By using the Cauchy product of power series and the definition of $q$-harmonic numbers, we can find that

$$
\sum_{n=1}^{\infty} \zeta_{n}\left[m, q^{p}\right] x^{n}=\frac{\operatorname{Li}_{m}\left[q^{p} x\right]}{1-x}, \quad|x|<1, m, p \in \mathbb{N} .
$$

Multiplying (2.11) by $x^{-1}-x^{k-1}$ and $q$-integrating over $(0, q)$ yield

$$
[k]_{q} \sum_{n=1}^{\infty} \frac{\zeta_{n}\left[m, q^{p}\right]}{[n]_{q}[n+k]_{q}} q^{n}=\operatorname{Li}_{m+1}\left[q^{p+1}\right]+\sum_{i=1}^{k-1} \sum_{n=1}^{\infty} \frac{q^{(p+1) n+i}}{[n]_{q}^{m}[n+i]_{q}},
$$

where the generalized $q$-integral is defined by $(a \leq x \neq \infty)($ see $[3,17,38,39])$

$$
\int_{a}^{x} f(t) d_{q} t=\int_{0}^{x} f(t) d_{q} t-\int_{0}^{a} f(t) d_{q} t=(1-q) \sum_{i=0}^{\infty} q^{i}\left[x f\left(q^{i} x\right)-a f\left(q^{i} a\right)\right]
$$

and

$$
\int_{0}^{x} f(t) d_{q} t=(1-q) x \sum_{i=0}^{\infty} q^{i} f\left(q^{i} x\right), \quad x \neq \infty
$$

Taking $p=m-1$ in (2.12) and $r=0$ in (1.12), we obtain

$$
\begin{aligned}
& {[k]_{q} \sum_{n=1}^{\infty} \frac{\zeta_{n}\left[m, q^{m-1}\right]}{[n]_{q}[n+k]_{q}} q^{n}=\mathrm{Li}_{m+1}\left[q^{m}\right]+\sum_{i=1}^{k-1} q^{i} \sum_{n=1}^{\infty} \frac{q^{m n}}{[n]_{q}^{m}[n+i]_{q}},} \\
& \sum_{n=1}^{\infty} \frac{q^{m n}}{[n]_{q}^{m}[n+k]_{q}}=\sum_{j=1}^{m-1} \frac{(-1)^{j-1}}{[k]_{q}^{j}} \operatorname{Li}_{m-j+1}\left[q^{m-j}\right]+(-1)^{m-1} \frac{\left[H_{k}^{(1)}\right]}{[k]_{q}^{m}} .
\end{aligned}
$$

Substituting (2.16) into (2.15) yields the desired result. We finish the proof of Theorem 2.3.

In fact, by a similar argument as in the proof of Theorem 2.3, we obtain the more general identity

$$
\begin{aligned}
{[k-r]_{q} \sum_{n=1}^{\infty} \frac{\zeta_{n}\left[m, q^{m-1}\right]}{[n+r]_{q}[n+k]_{q}} q^{n}=} & \sum_{j=1}^{m-1}(-1)^{j-1} \mathrm{Li}_{m-j+1}\left[q^{m-j}\right] \frac{\left[H_{k-1}^{(j)}\right]-\left[H_{r-1}^{(j)}\right]}{q^{r}} \\
& +(-1)^{m-1} \sum_{i=1}^{k-r} \frac{\left[H_{r+i-1}^{(1)}\right]}{[r+i-1]_{q}^{m}} q^{i-1}
\end{aligned}
$$


where $m, k, r \in \mathbb{N}$ and $r<k$. Putting $m=1$ and 2 in (2.10), we give the following two examples:

$$
\begin{aligned}
& \sum_{n=1}^{\infty} \frac{\left[H_{n}\right]}{[n]_{q}[n+k]_{q}} q^{n}=\frac{1}{[k]_{q}}\left\{\mathrm{Li}_{2}[q]+\frac{\left[H_{k}^{(1)}\right]^{2}+\zeta_{k}\left[2, q^{2}\right]}{2}-\frac{\left[H_{k}^{(1)}\right]}{[k]_{q}} q^{k}\right\} \\
& \sum_{n=1}^{\infty} \frac{\left[H_{n}^{(2)}\right]}{[n]_{q}[n+k]_{q}} q^{n}=\frac{1}{[k]_{q}}\left\{\operatorname{Li}_{3}\left[q^{2}\right]+\mathrm{Li}_{2}[q]\left[H_{k-1}^{(1)}\right]-\sum_{i=1}^{k-1} \frac{\left[H_{i}^{(1)}\right]}{[i]_{q}^{2}} q^{i}\right\}
\end{aligned}
$$

Corollary 2.4 For $m \in \mathbb{N}$, we have

$$
\begin{aligned}
\frac{1}{2} W_{0, m}^{(1,1)}[1,1 ; m+1]-(-1)^{m-1} W_{0,1}^{(0,1)}[1,1 ; m+1] \\
=W_{0, m+1}^{(1)}[1 ; m+2]+\sum_{j=1}^{m-1}(-1)^{j-1} \operatorname{Li}_{m-j+1}\left[q^{m-j}\right] W_{0,1}^{(0)}[1 ; j+1] \\
\quad-\frac{1}{2} W_{0, m}^{(2)}[2 ; m+1]-\operatorname{Li}_{2}[q] \mathrm{Li}_{m+1}\left[q^{m}\right]
\end{aligned}
$$

and

$$
\begin{aligned}
\frac{1}{2}\left\{W_{0, m}^{(0,1,1)}\left[\{1\}_{3} ; m+1\right]+W_{0, m}^{(0,2)}[1,2 ; m+1]\right\} \\
\quad-\frac{(-1)^{m-1}}{2}\left\{W_{0,1}^{(0,1,1)}\left[\{1\}_{3} ; m+1\right]+W_{0,1}^{(0,2)}[1,2 ; m+1]\right\} \\
=\left\{W_{0, m+1}^{(0,1)}[1,1 ; m+2]-(-1)^{m-1} W_{0,2}^{(0,1)}[1,1 ; m+2]\right\} \\
\quad-\mathrm{Li}_{2}[q]\left\{W_{0, m}^{(0)}[1 ; m+1]-(-1)^{m-1} W_{0,1}^{(0)}[1 ; m+1]\right\} \\
+\sum_{j=1}^{m-1}(-1)^{j-1} W_{0,1}^{(0)}[1 ; j+1] W_{0, m-j}^{(0)}[1 ; m-j+1] .
\end{aligned}
$$

Proof Formula (2.20) shows that multiplying (2.18) by $\frac{q^{m k}}{[k]_{q}^{m}}$ and summing with respect to $k$, then using (2.16) yield

$$
\begin{aligned}
& \sum_{n, k=1}^{\infty} \frac{\left[H_{n}\right]}{[k]_{q}^{m}[n]_{q}[n+k]_{q}} q^{n} q^{m k} \\
& \quad=\sum_{k=1}^{\infty} \frac{q^{m k}}{[k]_{q}^{m+1}}\left\{\operatorname{Li}_{2}[q]+\frac{\left[H_{k}^{(1)}\right]^{2}+\zeta_{k}\left[2, q^{2}\right]}{2}-\frac{\left[H_{k}^{(1)}\right]}{[k]_{q}} q^{k}\right\} \\
& \quad=\operatorname{Li}_{2}[q] \mathrm{Li}_{m+1}\left[q^{m}\right]+\frac{1}{2}\left\{W_{0, m}^{(1,1)}[1,1 ; m+1]+W_{0, m}^{(2)}[2 ; m+1]\right\}-W_{0, m+1}^{(1)}[1 ; m+2] \\
& \quad=\sum_{n=1}^{\infty} \frac{\left[H_{n}\right]}{[n]_{q}} q^{n} \sum_{k=1}^{\infty} \frac{q^{m k}}{[k]_{q}^{m}[n+k]_{q}} \\
& \quad=\sum_{n=1}^{\infty} \frac{\left[H_{n}\right]}{[n]_{q}} q^{n}\left\{\sum_{j=1}^{m-1} \frac{(-1)^{j-1}}{[n]_{q}^{j}} \operatorname{Li}_{m-j+1}\left[q^{m-j}\right]+(-1)^{m-1} \frac{\left[H_{n}^{(1)}\right]}{[n]_{q}^{m}}\right\}
\end{aligned}
$$




$$
\begin{aligned}
= & (-1)^{m-1} W_{0,1}^{(0,1)}[1,1 ; m+1] \\
& +\sum_{j=1}^{m-1}(-1)^{j-1} \mathrm{Li}_{m-j+1}\left[q^{m-j}\right] W_{0,1}^{(0)}[1 ; j+1] .
\end{aligned}
$$

By a direct calculation, we obtain the result (2.20).

Similarly, to prove (2.21), multiplying (2.18) by $\frac{\left[H_{k}\right]}{[k]_{q}^{m}} q^{m k}$ and summing with respect to $k$, then applying the same arguments as in the proof of (2.20), we may easily deduce the desired result.

Theorem 2.5 For $x, y \in[-1,1]$ and positive integers $m_{1}, m_{2}, k, r$ with $r<k$, the following identity holds:

$$
\begin{aligned}
{[k-r]_{q} } & \sum_{n=1}^{\infty} \frac{\zeta_{n}\left[m_{1}, x\right] \zeta_{n}\left[m_{2}, y\right]-\zeta_{n}\left[m_{1}+m_{2}, x y\right]}{[n+r]_{q}[n+k]_{q}} q^{n+r} \\
= & \sum_{i=1}^{k-r} \sum_{n=1}^{\infty}\left\{\frac{\zeta_{n}\left[m_{1}, x\right] y^{n}}{[n]_{q}^{m_{2}}[n+r+i-1]_{q}}+\frac{\zeta_{n}\left[m_{2}, y\right] x^{n}}{[n]_{q}^{m_{1}}[n+r+i-1]_{q}}\right. \\
& \left.-\frac{2(x y)^{n}}{[n]_{q}^{m_{1}+m_{2}}[n+r+i-1]_{q}}\right\} q^{n+r+i-1},
\end{aligned}
$$

where the partial sum $\zeta_{n}[m, x]$ is defined by

$$
\zeta_{n}[m, x]:=\sum_{j=1}^{n} \frac{x^{j}}{[j]_{q}^{m}}
$$

Proof To prove identity (2.23), we consider the generating function

$$
F[x, y, z]:=\sum_{n=1}^{\infty}\left\{\zeta_{n}\left[m_{1}, x\right] \zeta_{n}\left[m_{2}, y\right]-\zeta_{n}\left[m_{1}+m_{2}, x y\right]\right\} z^{n-1}, \quad z \in(-1,1) .
$$

By the definition of $\zeta_{n}[m, x]$, we can rewrite (2.24) as follows:

$$
\begin{aligned}
F[x, y, z] & =\sum_{n=1}^{\infty}\left\{\begin{array}{c}
\left(\zeta_{n}\left[m_{1}, x\right]+\frac{x^{n+1}}{[n+1]_{q}^{m_{1}}}\right)\left(\zeta_{n}\left[m_{2}, y\right]+\frac{x^{n+1}}{[n+1]_{q}^{m_{2}}}\right) \\
-\left(\zeta_{n}\left[m_{1}+m_{2}, x y\right]+\frac{x^{n+1} y^{n+1}}{[n+1]_{q}^{y_{1}+m_{2}}}\right)
\end{array}\right\} z^{n-1} \\
& =z F[x, y, z]+\sum_{n=1}^{\infty}\left\{\frac{\zeta_{n}\left[m_{1}, x\right]}{[n+1]_{q}^{m_{2}}} y^{n+1}+\frac{\zeta_{n}\left[m_{2}, y\right]}{[n+1]_{q}^{m_{1}}} x^{n+1}\right\} z^{n} \\
& =z F[x, y, z]+\sum_{n=1}^{\infty}\left\{\frac{\zeta_{n}\left[m_{1}, x\right]}{[n]_{q}^{m_{2}}} y^{n}+\frac{\zeta_{n}\left[m_{2}, y\right]}{[n]_{q}^{m_{1}}} x^{n}-2 \frac{x^{n} y^{n}}{[n]_{q}^{m_{1}+m_{2}}}\right\} z^{n-1} .
\end{aligned}
$$

Hence, we obtain the formula

$$
\begin{aligned}
& \sum_{n=1}^{\infty}\left\{\zeta_{n}\left[m_{1}, x\right] \zeta_{n}\left[m_{2}, y\right]-\zeta_{n}\left[m_{1}+m_{2}, x y\right]\right\} z^{n-1} \\
& \quad=\sum_{n=1}^{\infty}\left\{\frac{\zeta_{n}\left[m_{1}, x\right]}{[n]_{q}^{m_{2}}} y^{n}+\frac{\zeta_{n}\left[m_{2}, y\right]}{[n]_{q}^{m_{1}}} x^{n}-2 \frac{x^{n} y^{n}}{[n]_{q}^{m_{1}+m_{2}}}\right\} \frac{z^{n-1}}{1-z} .
\end{aligned}
$$


Multiplying (2.25) by $z^{r}-z^{k}$ and $q$-integrating over $(0, q)$, then using the identity

$$
\frac{q^{n+r}}{[n+r]_{q}}-\frac{q^{n+k}}{[n+k]_{q}}=[k-r]_{q} \frac{q^{n+r}}{[n+r]_{q}[n+k]_{q}},
$$

we can deduce (2.23). The proof is completed.

In fact, using the Cauchy product of power series, (2.25) can be rewritten as

$$
\begin{aligned}
& \sum_{n=1}^{\infty}\left\{\zeta_{n}\left[m_{1}, x\right] \zeta_{n}\left[m_{2}, y\right]-\zeta_{n}\left[m_{1}+m_{2}, x y\right]\right\} z^{n-1} \\
& \quad=\sum_{n=1}^{\infty} \sum_{k=1}^{n}\left\{\frac{\zeta_{k}\left[m_{1}, x\right]}{[k]_{q}^{m_{2}}} y^{k}+\frac{\zeta_{n}\left[m_{2}, y\right]}{[k]_{q}^{m_{1}}} x^{k}-2 \frac{x^{k} y^{k}}{[k]_{q}^{m_{1}+m_{2}}}\right\} z^{n-1} .
\end{aligned}
$$

Thus, comparing the coefficients of $z^{n-1}$ in (2.26), we obtain

$$
\sum_{k=1}^{n}\left\{\frac{\zeta_{k}\left[m_{1}, x\right]}{[k]_{q}^{m_{2}}} y^{k}+\frac{\zeta_{k}\left[m_{2}, y\right]}{[k]_{q}^{m_{1}}} x^{k}\right\}=\zeta_{n}\left[m_{1}, x\right] \zeta_{n}\left[m_{2}, y\right]+\zeta_{n}\left[m_{1}+m_{2}, x y\right]
$$

Similarly, considering the following function

$$
F[x, y]:=\sum_{n=1}^{\infty}\left\{\zeta_{n}^{3}[m, x]-3 \zeta_{n}[m, x] \zeta_{n}\left[2 m, x^{2}\right]+2 \zeta_{n}\left[3 m, x^{3}\right]\right\} y^{n-1}, \quad y \in(-1,1),
$$

by a similar argument as in the proof of (2.23), we deduce that

$$
\begin{aligned}
& \sum_{n=1}^{\infty}\left\{\zeta_{n}^{3}[m, x]-3 \zeta_{n}[m, x] \zeta_{n}\left[2 m, x^{2}\right]+2 \zeta_{n}\left[3 m, x^{3}\right]\right\} y^{n-1} \\
& \quad=3 \sum_{n=1}^{\infty}\left\{\frac{\zeta_{n}^{2}[m, x]}{[n]_{q}^{m}} x^{n}-\frac{\zeta_{n}\left[2 m, x^{2}\right]}{[n]_{q}^{m}} x^{n}-2 \frac{\zeta_{n}[m, x]}{[n]_{q}^{2 m}} x^{2 n}+2 \frac{x^{3 n}}{[n]_{q}^{3 m}}\right\} \frac{y^{n-1}}{1-y}
\end{aligned}
$$

By using the Cauchy product of power series again and then comparing the coefficients of $y^{n-1}$, we obtain

$$
\begin{gathered}
3 \sum_{k=1}^{n}\left\{\frac{\zeta_{k}^{2}[m, x]-\zeta_{k}\left[2 m, x^{2}\right]}{[k]_{q}^{m}} x^{k}-2 \frac{\zeta_{k}[m, x]}{[k]_{q}^{2 m}} x^{2 k}+2 \frac{x^{3 k}}{[k]_{q}^{3 m}}\right\} \\
=\zeta_{n}^{3}[m, x]-3 \zeta_{n}[m, x] \zeta_{n}\left[2 m, x^{2}\right]+2 \zeta_{n}\left[3 m, x^{3}\right] .
\end{gathered}
$$

Combining (2.27) and (2.29), we have the result

$$
\sum_{k=1}^{n} \frac{\zeta_{k}^{2}[m, x]+\zeta_{k}\left[2 m, x^{2}\right]}{[k]_{q}^{m}} x^{k}=\frac{1}{3}\left\{\zeta_{n}^{3}[m, x]+3 \zeta_{n}[m, x] \zeta_{n}\left[2 m, x^{2}\right]+2 \zeta_{n}\left[3 m, x^{3}\right]\right\}
$$

Setting $x=q, m=1$ in the above equation, we obtain

$$
\sum_{k=1}^{n} \frac{\left[H_{k}^{(1)}\right]^{2}+\zeta_{k}\left[2, q^{2}\right]}{[k]_{q}} q^{k}=\frac{1}{3}\left\{\left[H_{n}^{(1)}\right]^{3}+3\left[H_{n}^{(1)}\right] \zeta_{n}\left[2, q^{2}\right]+2 \zeta_{n}\left[3, q^{3}\right]\right\} .
$$


Theorem 2.6 For positive integers $m, r, k$ with $r<k$ and $x \in[-1,1]$, the following identity holds:

$$
\begin{aligned}
& {[k-r]_{q} \sum_{n=1}^{\infty} \frac{\zeta_{n}^{3}[m, x]-\zeta_{n}\left[3 m, x^{3}\right]}{[n+r]_{q}[n+k]_{q}} q^{n+r}} \\
& \quad=3 \sum_{i=1}^{k-r} \sum_{n=1}^{\infty}\left\{\frac{\zeta_{n}^{2}[m, x]}{[n]_{q}^{m}[n+r+i-1]_{q}} x^{n}-\frac{\zeta_{n}[m, x]}{[n]_{q}^{2 m}[n+r+i-1]_{q}} x^{2 n}\right\} q^{n+r+i-1} .
\end{aligned}
$$

Proof Similar as in the proof of Theorem 2.5, we consider the power series

$$
F[x, y]:=\sum_{n=1}^{\infty}\left\{\zeta_{n}^{3}[m, x]-\zeta_{n}\left[3 m, x^{3}\right]\right\} y^{n-1}, \quad y \in(-1,1)
$$

and apply the same arguments as in the proof of Theorem 2.5. We may deduce formula (2.31).

Taking $r=0$ and $m_{1}=m_{2}=x=y=1$ in (2.23), we get

$$
\begin{aligned}
{[k]_{q} } & \sum_{n=1}^{\infty} \frac{\left[H_{n}\right]^{2}-\zeta_{n}[2]}{[n]_{q}[n+k]_{q}} q^{n} \\
= & 2 \sum_{i=1}^{k-1} q^{i} \sum_{n=1}^{\infty}\left\{\frac{\left[H_{n}\right]}{[n]_{q}[n+i]_{q}} q^{n}-\frac{q^{n}}{[n]_{q}^{2}[n+i]_{q}}\right\}+2 \sum_{n=1}^{\infty}\left\{\frac{\left[H_{n}\right]}{[n]_{q}^{2}} q^{n}-\frac{q^{n}}{[n]_{q}^{3}}\right\} \\
= & 2 \mathrm{Li}_{3}\left[q^{2}\right]+2 \mathrm{Li}_{2}[q]\left[H_{k-1}^{(1)}\right]+\sum_{i=1}^{k-1} \frac{\left[H_{i}^{(1)}\right]^{2}+\zeta_{i}\left[2, q^{2}\right]}{[i]_{q}} q^{i} \\
& -2 \sum_{i=1}^{k-1} \frac{\left[H_{i}^{(1)}\right]}{[i]_{q}^{2}} q^{2 i}-2 \sum_{i=1}^{k-1} q^{i} \sum_{n=1}^{\infty} \frac{q^{n}}{[n]_{q}^{2}[n+i]_{q}} .
\end{aligned}
$$

From (1.17) and (2.19), we have

$$
\begin{aligned}
{[k]_{q} \sum_{n=1}^{\infty} \frac{\zeta_{n}[2]}{[n]_{q}[n+k]_{q}} q^{n}=} & {[k]_{q} \sum_{n=1}^{\infty} \frac{\left[H_{n}^{(2)}\right]}{[n]_{q}[n+k]_{q}} q^{n}+(1-q)[k]_{q} \sum_{n=1}^{\infty} \frac{\left[H_{n}\right]}{[n]_{q}[n+k]_{q}} q^{n} } \\
= & \operatorname{Li}_{3}\left[q^{2}\right]+\mathrm{Li}_{2}[q]\left[H_{k-1}^{(1)}\right]-\sum_{i=1}^{k-1} \frac{\left[H_{i}^{(1)}\right]}{[i]_{q}^{2}} q^{i} \\
& +(1-q)\left\{\mathrm{Li}_{2}[q]+\frac{\left[H_{k}^{(1)}\right]^{2}+\zeta_{k}\left[2, q^{2}\right]}{2}-\frac{\left[H_{k}^{(1)}\right]}{[k]_{q}} q^{k}\right\} .
\end{aligned}
$$

Substituting (2.33) into (2.32) results in

$$
\begin{aligned}
& {[k]_{q} \sum_{n=1}^{\infty} \frac{\left[H_{n}\right]^{2}}{[n]_{q}[n+k]_{q}} q^{n}} \\
& \quad=3 \mathrm{Li}_{3}\left[q^{2}\right]+3 \mathrm{Li}_{2}[q]\left[H_{k-1}^{(1)}\right]+\sum_{i=1}^{k-1} \frac{\left[H_{i}^{(1)}\right]^{2}+\zeta_{i}\left[2, q^{2}\right]}{[i]_{q}} q^{i}
\end{aligned}
$$




$$
\begin{aligned}
& -2 \sum_{i=1}^{k-1} \frac{\left[H_{i}^{(1)}\right]}{[i]_{q}^{2}} q^{2 i}-\sum_{i=1}^{k-1} \frac{\left[H_{i}^{(1)}\right]}{[i]_{q}^{2}} q^{i}-2 \sum_{i=1}^{k-1} q^{i} \sum_{n=1}^{\infty} \frac{q^{n}}{[n]_{q}^{2}[n+i]_{q}} \\
& +(1-q)\left\{\mathrm{Li}_{2}[q]+\frac{\left[H_{k}^{(1)}\right]^{2}+\zeta_{k}\left[2, q^{2}\right]}{2}-\frac{\left[H_{k}^{(1)}\right]}{[k]_{q}} q^{k}\right\} .
\end{aligned}
$$

Similarly, putting $r=0, m=1, x=1$ in (2.31), we get

$$
\begin{aligned}
{[k]_{q} \sum_{n=1}^{\infty} \frac{\left[H_{n}\right]^{3}-\zeta_{n}[3]}{[n]_{q}[n+k]_{q}} q^{n}=} & 3 \sum_{n=1}^{\infty}\left\{\frac{\left[H_{n}\right]^{2}}{[n]_{q}^{2}} q^{n}-\frac{\left[H_{n}\right]}{[n]_{q}^{3}} q^{n}\right\} \\
& +3 \sum_{i=1}^{k-1} q^{i} \sum_{n=1}^{\infty}\left\{\frac{\left[H_{n}\right]^{2}}{[n]_{q}[n+i]_{q}}-\frac{\left[H_{n}\right]}{[n]_{q}^{2}[n+i]_{q}}\right\} q^{n}
\end{aligned}
$$

From (2.10), (2.34) and (2.35), we know that the cubic $q$-sums

$$
\sum_{n=1}^{\infty} \frac{\left[H_{n}\right]^{3}}{[n]_{q}[n+k]_{q}} q^{n}
$$

are reducible to $q$-polylogarithms, $q$-harmonic numbers and $q$-rational series. Letting $q$ tend to 1 in (2.34), we get the expression for quadratic sums

$$
\sum_{n=1}^{\infty} \frac{H_{n}^{2}}{n(n+k)}
$$

in terms of harmonic numbers and zeta values:

$$
\sum_{n=1}^{\infty} \frac{H_{n}^{2}}{n(n+k)}=\frac{1}{k}\left\{\begin{array}{c}
3 \zeta(3)+\frac{H_{k}^{3}+3 H_{k} H_{k}^{(2)}+2 H_{k}^{(3)}}{3} \\
-\frac{H_{k}^{2}+H_{k}^{(2)}}{k}-\sum_{i=1}^{k-1} \frac{H_{i}}{i^{2}}+\zeta(2) H_{k-1}
\end{array}\right\} .
$$

Note that result (2.36) is given in Sofo's paper [40] and Xu's paper [1] with Zhang and Zhu. It should be emphasized that the papers $[1,2,4,5,40]$ also contain many other types of results. For example, from [1], we have the result

$$
\sum_{n=1}^{\infty} \frac{H_{n}^{3}}{n(n+k)}=\frac{1}{k}\left\{\begin{array}{l}
10 \zeta(4)+\frac{H_{k}^{4}+8 H_{k} H_{k}^{(3)}+6 H_{k}^{2} H_{k}^{(2)}+3\left(H_{k}^{(2)}\right)^{2}+6 H_{k}^{(4)}}{4} \\
\quad-\frac{H_{k}^{3}+3 H_{k} H_{k}^{(2)}+2 H_{k}^{(3)}}{k}+4 \zeta(3) H_{k-1}+\frac{1}{2} \zeta(2) H_{k-1}^{(2)} \\
\quad+\frac{3}{2} \zeta(2) H_{k-1}^{2}+\sum_{i=1}^{k-1} \frac{H_{i}}{i^{3}}-\frac{3}{2} \sum_{i=1}^{k-1} \frac{H_{i}^{2}+H_{i}^{(2)}}{i^{2}}-3 \sum_{i=1}^{k-1} \frac{1}{i} \sum_{j=1}^{i} \frac{H_{j}}{j^{2}}
\end{array}\right\}
$$

The result above can also be obtained by using (2.35) with letting $q \rightarrow 1$.

\section{Some expressions of series involving $q$-harmonic numbers and $q$-binomial coefficients}

In this section, we give some closed form sums of $W_{k, t}^{(\mathbf{s})}[\mathbf{m} ; p]$ through $q$-polylogarithms, $q$-harmonic numbers and other $q$-series. 
From (2.8) and (2.9), we have the expansions

$$
\begin{aligned}
& W_{k, k+p-1}^{\left(s_{1}, \ldots, s_{r}\right)}\left[m_{1}, m_{2}, \ldots, m_{r} ; p\right] \\
& =\sum_{i=1}^{k}(-1)^{i-1}[i]_{q}\left[\begin{array}{c}
k \\
i
\end{array}\right]_{q} q^{-\frac{i}{2}(2 k-1-i)} \\
& \quad \times \sum_{n=1}^{\infty} \frac{\zeta_{n}\left[m_{1}, q^{s_{1}}\right] \zeta_{n}\left[m_{2}, q^{s_{2}}\right] \cdots \zeta_{n}\left[m_{r}, q^{s_{r}}\right]}{[n]_{q}^{p}[n+i]_{q}} q^{p n}
\end{aligned}
$$

and

$$
\begin{aligned}
& W_{k, k-1}^{\left(s_{1}, \ldots, s_{r}\right)}\left[m_{1}, m_{2}, \ldots, m_{r} ; 0\right] \\
& =[k]_{q} \sum_{i=1}^{k-1}(-1)^{i-1}[i]_{q}\left[\begin{array}{c}
k-1 \\
i
\end{array}\right]_{q} q^{(2-k)-\frac{i}{2}(2 k-3-i)} \\
& \quad \times \sum_{n=1}^{\infty} \frac{\zeta_{n}\left[m_{1}, q^{s_{1}}\right] \zeta_{n}\left[m_{2}, q^{s_{2}}\right] \cdots \zeta_{n}\left[m_{r}, q^{s_{r}}\right]}{[n+1]_{q}[n+1+i]_{q}} q^{n} .
\end{aligned}
$$

Hence, combining formulas (2.10), (2.17), (2.34), (3.1) and (3.2), by direct calculations, we can get the following three results.

Theorem 3.1 For positive integers $m, k$ and $p$,

$$
\begin{aligned}
& W_{k, k+p-1}^{(m-1)}[m ; p] \\
& =\sum_{i=1}^{k}(-1)^{i-1}[i]_{q}\left[\begin{array}{c}
k \\
i
\end{array}\right]_{q} q^{-\frac{i}{2}(2 k-1-i)} \sum_{n=1}^{\infty} \frac{\zeta_{n}\left[m, q^{m-1}\right]}{[n]_{q}^{p}[n+i]_{q}} q^{p n} \\
& =\sum_{i=1}^{k}(-1)^{i-1}[i]_{q}\left[\begin{array}{c}
k \\
i
\end{array}\right]_{q} q^{-\frac{i}{2}(2 k-1-i)}\left\{\sum_{j=1}^{p-1} \frac{(-1)^{j-1}}{[i]_{q}^{j}} \sum_{n=1}^{\infty} \frac{\zeta_{n}\left[m, q^{m-1}\right]}{[n]_{q}^{p+1-j}} q^{(p-j) n}\right\} \\
& +\sum_{i=1}^{k}(-1)^{i-1}\left[\begin{array}{c}
k \\
i
\end{array}\right]_{q} q^{-\frac{i}{2}(2 k-1-i)} \\
& \quad \times \frac{(-1)^{p-1}}{[i]_{q}^{p-1}}\left\{\begin{array}{c}
\mathrm{Li}_{m+1}\left[q^{m}\right]+(-1)^{m-1} \sum_{j=1}^{i-1} \frac{\left[H_{j}^{(1)}\right]}{[j]_{q}^{m}} q^{j} \\
+\sum_{j=1}^{m-1}(-1)^{j-1} \mathrm{Li}_{m+1-j}\left[q^{m-j}\right]\left[H_{i-1}^{(j)}\right]
\end{array}\right\}
\end{aligned}
$$

Theorem 3.2 For positive integers $m$ and $k$,

$$
\begin{aligned}
& W_{k, k-1}^{(m-1)}[m ; 0] \\
& \quad=[k]_{q} \sum_{i=1}^{k-1}(-1)^{i-1}[i]_{q}\left[\begin{array}{c}
k-1 \\
i
\end{array}\right]_{q} q^{(2-k)-\frac{i}{2}(2 k-3-i)} \sum_{n=1}^{\infty} \frac{\zeta_{n}\left[m, q^{m-1}\right]}{[n+1]_{q}[n+1+i]_{q}} q^{n} \\
& \quad=[k]_{q} \sum_{i=1}^{k-1}(-1)^{i-1}\left[\begin{array}{c}
k-1 \\
i
\end{array}\right]_{q} q^{(2-k)-\frac{i}{2}(2 k-3-i)}\left\{\begin{array}{c}
\sum_{j=1}^{m-1}(-1)^{j-1} \operatorname{Li}_{m-j+1}\left[q^{m-j}\right] \frac{\left[H_{i}^{(j)}\right]}{q} \\
+(-1)^{m-1} \sum_{j=1}^{i} \frac{\left[H_{j}^{(1)}\right]}{[j]_{q}^{m}} q^{j-1}
\end{array}\right\} .
\end{aligned}
$$


Theorem 3.3 For positive integer $k$,

$$
\begin{aligned}
& W_{k, k}^{(0,0)}[1,1 ; 1] \\
& =\sum_{i=1}^{k}(-1)^{i-1}[i]_{q}\left[\begin{array}{c}
k \\
i
\end{array}\right]_{q} q^{-\frac{i}{2}(2 k-1-i)} \sum_{n=1}^{\infty} \frac{\left[H_{n}\right]^{2}}{[n]_{q}[n+i]_{q}} q^{n} \\
& =\sum_{i=1}^{k}(-1)^{i-1}\left[\begin{array}{c}
k \\
i
\end{array} q_{q}^{-\frac{i}{2}(2 k-1-i)}\left\{\begin{array}{c}
3 \mathrm{Li}_{3}\left[q^{2}\right]+3 \mathrm{Li}_{2}[q]\left[H_{i-1}^{(1)}\right] \\
+\sum_{j=1}^{i-1} \frac{\left[H_{j}^{(1)}\right]^{2}+\zeta_{j}\left[2, q^{2}\right]}{[j]_{q}} q^{j} \\
-2 \sum_{j=1}^{i-1} \frac{\left[H_{j}^{(1)}\right]}{[j]_{q}^{2}} q^{2 j}-\sum_{j=1}^{i-1} \frac{\left[H_{j}^{(1)}\right]}{[j]_{q}^{2}} q^{j} \\
-2 \sum_{j=1}^{i-1} q^{j} \sum_{n=1}^{\infty} \frac{q^{n}}{[n]_{q}^{2}[n+j]_{q}}+(1-q) \mathrm{Li}_{2}[q] \\
+(1-q)\left(\frac{\left[H_{i}^{(1)}\right]^{2}+\zeta_{i}\left[2, q^{2}\right]}{2}-\frac{\left[H_{i}^{(1)}\right]}{[i]_{q}} q^{i}\right)
\end{array}\right\} .\right.
\end{aligned}
$$

Taking $p=1$ in (3.3), we have

$$
\begin{aligned}
& W_{k, k}^{(m-1)}[m ; 1] \\
& \quad=\sum_{i=1}^{k}(-1)^{i-1}\left[\begin{array}{c}
k \\
i
\end{array}\right]_{q} q^{-\frac{i}{2}(2 k-1-i)}\left\{\begin{array}{c}
\operatorname{Li}_{m+1}\left[q^{m}\right]+(-1)^{m-1} \sum_{j=1}^{i-1} \frac{\left[H_{j}^{(1)}\right]}{[j]_{q}^{m}} q^{j} \\
+\sum_{j=1}^{m-1}(-1)^{j-1} \mathrm{Li}_{m+1-j}\left[q^{m-j}\right]\left[H_{i-1}^{(j)}\right]
\end{array}\right\} .
\end{aligned}
$$

Moreover, from (2.10), (2.34), (2.35) and (3.1), we know that the cubic $q$-Euler type sum $W_{k, k}^{(0,0,0)}\left[\{1\}_{3} ; 1\right]$ is reducible to $q$-polylogarithms, $q$-harmonic numbers and $q$-rational series. Letting $q \rightarrow 1$ in (3.5) and (3.6), we obtain the well-known identities [1]

$$
\begin{aligned}
& W_{k}(m ; 0)=k \sum_{i=1}^{k-1}(-1)^{i-1}\left(\begin{array}{c}
k-1 \\
i
\end{array}\right)\left\{\sum_{j=1}^{m-1}(-1)^{j-1} \zeta(m-j+1) H_{i}^{(j)}+(-1)^{m-1} \sum_{j=1}^{i} \frac{H_{j}}{j^{m}}\right\}, \\
& W_{k}(m ; 1)=\sum_{r=1}^{k}(-1)^{r+1}\left(\begin{array}{c}
k \\
r
\end{array}\right)\left\{\begin{array}{c}
\zeta(m+1)+(-1)^{m-1} \sum_{i=1}^{r-1} \frac{H_{i}}{i^{m}} \\
+\sum_{j=1}^{m-1}(-1)^{j-1} \zeta(m+1-j) H_{r-1}^{(j)}
\end{array}\right\}, \\
& W_{k}\left(\{1\}_{2} ; 1\right)=\sum_{r=1}^{k}(-1)^{r+1}\left(\begin{array}{c}
k \\
r
\end{array}\right)\left\{\begin{array}{c}
3 \zeta(3)+\frac{H_{r}^{3}+3 H_{r} H_{r}^{(2)}+2 H_{r}^{(3)}}{3} \\
-\frac{H_{r}^{2}+H_{r}^{(2)}}{r}-\sum_{i=1}^{r-1} \frac{H_{i}}{i^{2}}+\zeta(2) H_{r-1}
\end{array}\right\} .
\end{aligned}
$$

Acknowledgements

The author is most grateful to two referees for helpful comments and suggestions.

Competing interests

The author declares that they have no competing interests.

Authors' contributions

The author read and approved the final manuscript.

\section{Publisher's Note}

Springer Nature remains neutral with regard to jurisdictional claims in published maps and institutional affiliations.

Received: 2 October 2017 Accepted: 7 January 2018 Published online: 18 January 2018

\section{References}

1. Xu, C, Zhang, M, Zhu, W: Some evaluation of harmonic number sums. Integral Transforms Spec. Funct. 27(12), 937-955 (2016) 
2. Sofo, A: Harmonic sums and integral representations. J. Appl. Anal. 16, 265-277 (2010)

3. Andrews, GE, Askey, R, Roy, R: Special Functions, pp. 481-532. Cambridge University Press, Cambridge (2000)

4. Sofo, A: Quadratic alternating harmonic number sums. J. Number Theory 154, 144-159 (2015)

5. Sofo, A, Srivastava, HM: Identities for the harmonic numbers and binomial coefficients. Ramanujan J. 25, 93-113 (2011)

6. Sofo, A: Shifted harmonic sums of order two. Commun. Korean Math. Soc. 29(2), 239-255 (2014)

7. Flajolet, P, Salvy, B: Euler sums and contour integral representations. Exp. Math. 7(1), 15-35 (1998)

8. Bailey, DH, Borwein, JM, Girgensohn, R: Experimental evaluation of Euler sums. Exp. Math. 3(1), 17-30 (1994)

9. Borwein, JM, Bradley, DM: Thirty-two Goldbach variations. Int. J. Number Theory 2(1), 65-103 (2006)

10. Borwein, D, Borwein, JM, Bradley, DM: Parametric Euler sum identities. J. Math. Anal. Appl. 316(1), 328-338 (2008)

11. Borwein, D, Borwein, JM, Girgensohn, R: Explicit evaluation of Euler sums. Proc. Edinburgh Math. 38, 277-294 (1995)

12. Borwein, J, Borwein, $P$, Girgensohn, R, Parnes, S: Making sense of experimental mathematics. Math. Intelligencer 18(4), 12-18 (1996)

13. Borwein, JM, Zucker, IJ, Boersma, J: The evaluation of character Euler double sums. Ramanujan J. 15(3), 377-405 (2008)

14. Freitas, P: Integrals of polylogarithmic functions, recurrence relations, and associated Euler sums. Math. Comp. 74(251), 1425-1440 (2005)

15. Xu, C, Yan, Y, Shi, Z: Euler sums and integrals of polylogarithm functions. J. Number Theory 165, 84-108 (2016)

16. $\mathrm{Xu}, \mathrm{C}, \mathrm{Li}, \mathrm{Z}$ : Tornheim type series and nonlinear Euler sums. J. Number Theory 174, 40-67 (2017)

17. Xu, C, Zhang, M, Zhu, W: Some evaluation of q-analogues of Euler sums. Monatsh. Math. 182(4), 957-975 (2017)

18. Dilcher, K, Pilehrood, KH, Pilehrood, TH: On q-analogues of double Euler sums. J. Math. Anal. Appl. 2(410), 979-988 (2014)

19. Borwein, JM, Girgensohn, R: Evaluation of triple Euler sums. Electron. J. Combin. 3(1), 2-7 (1996)

20. Choi, J: Finite summation formulas involving binomial coefficients, harmonic numbers and generalized harmonic numbers. J. Inequal. Appl. 2013, 49 (2013)

21. Li, Z, Xu, C: On q-analogues of quadratic Euler sums (2017) arXiv:1702.08507v2

22. Hessami Pilehrood, K, Hessami Pilehrood, T: On $q$-analogues of two-one formulas for multiple harmonic sums and multiple zeta star values. Monatsh. Math. 176, 275-291 (2015)

23. Kim, DS, Kim, T: Identities involving harmonic and hyperharmonic numbers. Adv. Difference Equ. 235, 1-15 (2013)

24. Kim, T: On p-adic q-L-functions and sums of powers. Discrete Math. 252(1-3), 179-187 (2002)

25. Kim, T: On Euler-Barnes multiple zeta functions. Russ. J. Math. Phys. 10(3), 261-267 (2003)

26. Kim, T: A note on the q-multiple zeta function. Adv. Stud. Contemp. Math. (Kyungshang) 8(2), 111-113 (2004)

27. Kim, T: q-Riemann zeta function. Int. J. Math. Math. Sci. 12(9), 599-605 (2004)

28. Kim, T: Analytic continuation of multiple $q$-zeta functions and their values at negative integers. Russ. J. Math. Phys. 11(1), 71-76 (2004)

29. Kim, T: Barnes-type multiple q-zeta functions and q -Euler polynomials. J. Phys. A 43(25), 201-255 (2010)

30. Kim, T: A family of $(h, q)$-zeta function associated with $(h, q)$-Bernoulli numbers and polynomials. J. Comput. Anal. Appl. 14(3), 402-409 (2012)

31. Kizilates, C, Tuglu, N: Some combinatorial identities of $q$-harmonic and $q$-hyperharmonic numbers. Commun. Math. Appl. 6(2), 33-40 (2015)

32. Lorente, AS: Some $q$-representations of the $q$-analogue of the Hurwitz zeta function. Lect. Mat. 36(1), 13-20 (2015)

33. Srivastava, HM, Kim, T, Simsek, Y: q-Bernoulli numbers and polynomials associated with multiple $q$-zeta functions and basic L-series. Russ. J. Math. Phys. 12(3), 241-268 (2005)

34. Salem, A: Two classes of bounds for the $q$-gamma and the $q$-digamma functions in terms of the $q$-zeta functions. Banach J. Math. Anal. 8(1), 109-117 (2014)

35. Tomita, Y: Hermite's formulas for $q$-analogues of Hurwitz zeta functions. Funct. Approx. Comment. Math. 45(2), 289-301 (2011)

36. Wakayama, M, Yamasaki, Y: Integral representations of $q$-analogues of the Hurwitz zeta function. Monatsh. Math. 149(2), 141-154 (2006)

37. Zhao, J: q-multiple zeta functions and q-multiple polylogarithms. Ramanujan J. 14(2), 189-221 (2003)

38. Annaby, MH, Mansour, ZS: q-Fractional Calculus and Equations. Springer, Heidelberg (2012)

39. Jackson, HF: q-difference equations. Amer. J. Math. 32, 305-314 (1910)

40. Sofo, A: Mixed binomial sum identities. Integral Transforms Spec. Funct. 24(3), 187-200 (2013)

\section{Submit your manuscript to a SpringerOpen ${ }^{\circ}$ journal and benefit from:}

- Convenient online submission

- Rigorous peer review

- Open access: articles freely available online

- High visibility within the field

- Retaining the copyright to your article

Submit your next manuscript at $\gg$ springeropen.com 